\title{
Correspondence
}

\section{Recruitment crisis - poor marketing or product failure?}

I was interested to read Sekhri \& Sibbett's expansion on the topic of recruitment problems in psychiatry. They made a number of valid points and I look forward to the results of their proposed study of the opinions of foundation trainees. I was rather disappointed to see their letter conclude on the familiar note that the problem lies in a failure of marketing, or an 'underselling [of] psychiatry', which also seems to be the line taken by the Royal College of Psychiatrists with their campaign to promote psychiatry to medical and other students. ${ }^{2}$ This is not a new approach. Instead of concluding that the problem lies in not getting the message out, should psychiatrists not be listening to the message coming in from trainees in the falling recruitment numbers, and overhauling the specialty?

Of the many factors discussed, the negative view of psychiatry from other medical professionals and other sectors of society such as the media surely has a corrosive effect on recruitment. A 'zero tolerance' approach to stigma has recently been proposed by the College, ${ }^{3}$ although it remains to be seen how effective this will be at counteracting perceptions of psychiatry as a 'Cinderella specialty'. As Sekhri \& Sibbett state, the 'separatedness' of psychiatry is likely compounded by the structural changes to health services. Most mental health services are run out of separate hospitals, and indeed separate trusts, from other medical specialties. In the post-asylum era of acute care it is not clear why this is of benefit to either patients or psychiatrists. One does not need to be a psychiatrist to know that stigma feeds on perceptions of separateness. Medical students and other doctors rarely see psychiatrists in grand rounds, in the doctors' mess or making rounds on other wards. Should we not now review whether such enforced separation of medical management of mental illness from medical management of physical illness is still justified?

There is general hope that more exposure to psychiatry during the foundation programme will increase the attractiveness of the specialty. We should also consider that the opposite may be true. The 'multidisciplinary' approach has taken a form and function in mental health such that it is now debatable whether even a consultant psychiatrist is the leader of clinical care, to a far greater degree than surgical or medical counterparts. There have been numerous reports extolling the demedicalisation of psychiatry ${ }^{4}$ and a deskilling in fundamental aspects of psychiatric care such as psychopharmacology. ${ }^{5}$ Also, whereas Sekhri \& Sibbett draw attention to the heavy reliance of psychiatry on international medical graduates, a review of the pass marks for the College membership exams makes one wonder whether psychiatry has been successful in attracting doctors with the necessary linguistic, academic and clinical qualities required by such a demanding specialty. Accepting suboptimal candidates into psychiatry posts to maintain numbers may not be in the specialty's long-term interests, any more than it is in patients' interests. All of these factors may lead to a negative response from interested trainees on further exposure to the clinical realities of psychiatry.
A lack of sales is not always due to a failure of selling but can be caused by defects in the product itself. Rather than embarking on yet another marketing campaign, is it not time for the profession to listen to what trainees are saying and remake itself as a medical specialty fit for the 21 st century?

1 Sekhri R, Sibbett R. Recruitment in psychiatry: a complex and multifactorial problem. Psychiatrist 2012; 36: 118-9.

2 Psychiatry's identity crisis. Lancet 2012; 379: 1274.

3 Jaques $\mathrm{H}$. Royal college launches five year plan to increase applicants to psychiatry. BMJ Careers 2012; 27 Mar.

4 Lennox BR, Coles AJ, Vincent A. Antibody-mediated encephalitis: a treatable cause of schizophrenia. Br J Psychiatry 2012; 200: 92-4.

5 Harrison PJ, Baldwin DS, Barnes TRE, Burns T, Ebmeier KP, Ferrier IN, et al. No psychiatry without psychopharmacology. Br J Psychiatry 2011; 199: $263-5$.

Jonathan D. Hafferty, Core Trainee, Oxford Deanery, Oxford, UK, email: jonathanhafferty@gmail.com

doi: 10.1192/pb.36.7.271

\section{Medical students' views of psychiatric teaching methods}

Simmons \& Wilkinson ${ }^{1}$ demonstrated that medical students found cased-based discussion of child psychiatry more enjoyable and engaging than didactic lectures, with no reduction in exam performance. As the authors note, there is a dearth of studies comparing students' experience and enjoyment of different teaching methods in psychiatry. We welcome research of this nature as it may help us to improve the undergraduate experience of medical students in psychiatry.

We conducted a survey of two cohorts of students' $(n=38)$ experiences of a 12-week undergraduate psychiatry rotation at a London teaching hospital. The programme consisted of grand rounds, in which students presented a case to the rest of the cohort and were marked by senior psychiatric trainees; web-based scenarios - online, problem-based cases with associated questions which students completed themselves and then were taught around the topic by junior psychiatric trainees; a series of seminars delivered by consultants and senior trainees; and firm clinical teaching including weekly tutorials by consultants.

The survey showed that on a range of 1 (very poor) to 5 (excellent), grand rounds received the highest average rating (4.1), followed by web-based scenarios (3.9), seminars (3.7) and finally firm teaching (3.6). Free-text responses showed that incorporating role-play style teaching into sessions was seen as particularly useful and students wanted more teaching delivered in this way. There was considerable variation in students' experience of firm teaching, with some commenting on the lack of clinical experience or poor-quality tutorials, and others requesting more teaching with junior psychiatric trainees. Clinical teaching by its very nature is difficult to standardise as patients may not attend appointments and clinicians will have differing degrees of aptitude and 